\title{
Overshadowing of situational cues with variable but not fixed intertrial intervals
}

\author{
VINCENT M. LOLORDO \\ Dalhousie University, Halifax, Nova Scotia, Canada \\ DOUGLAS A. WILLIAMS \\ University of Winnipeg, Winnipeg, Manitoba, Canada \\ and \\ JANICE E. MCPHEE \\ Florida Gulf Coast University, Fort Myers, Florida
}

\begin{abstract}
In two experiments, we examined the conditions under which signaling an unconditioned stimulus (US) with a nominal conditioned stimulus (CS) interferes with the conditioning of situational cues in defensive freezing in the rat. Subjects received footshock USs that were (1) either signaled or unsignaled and (2) either varied or fixed in their temporal location within the conditioning session. Experiment 1 , with only one trial per session, yielded no evidence that signaling affected pretrial freezing using either a fixed or variable interval between placement in the context and shock onset. In a test in which no CSs or footshocks were presented, groups that previously had received footshock at a fixed temporal location showed greatest freezing at around that same time. For groups that had received footshocks at various times, freezing declined across the test session. Experiment 2 showed overshadowing of pretrial freezing after more extensive conditioning with many trials per session, but only if the intershock intervals were variable rather than fixed.
\end{abstract}

One of the most contentious aspects of the RescorlaWagner model (Rescorla \& Wagner, 1972; Wagner \& Rescorla, 1972) has been its assertion that contexts and conditioned stimuli (CSs) compete for association with the unconditioned stimulus (US). This assertion has inspired a large amount of empirical research assessing competitive interaction setween contex ts and nominal CSs (e.g., Balsam \& Tomie, 1985) and has stimulated the development of models that hold the antithesis - the assertion that learning about contexts and CSs occurs independently (e.g., Gibbon \& Balsam, 1981; Jenkins, Barnes, \& Barrera, 1981; Miller \& Schachtman, 1985).

One class of experiments assessing whether contexts and nominal CSs compete for behavioral control examines whether contextual conditioning can be overshadowed by a well-conditioned CS. In a typical experiment, USs are administered to two groups of subjects. In the signaled group, each US is preceded by a CS; in the control group the USs are unsignaled. At issue is whether there will be weaker contextual conditioning in the signaled group than in the unsignaled group, as predicted by the Rescorla-Wagner model.

This work was supported by grants from the Natural Sciences and Engineering Council of Canada. Correspondence should be directed to V. M. LoLordo, Department of Psychology, Dalhousie University, Halifax, NS, B3H 4J1, Canada (e-mail: vinlolo@is.dal.ca).
Experiments in this class have produced mixed outcomes. Baker, Mercier, Gabel, and Baker (1981, Experiment 4) reported that fear conditioning to the experimental context was attenuated if the shock US was signaled by a discrete CS. Groups of rats were rewarded for pressing a lever, and then in the absence of the lever they received six sessions in which eight footshocks occurred at variable intervals. For half the rats the footshocks were unsignaled, and for the other rats each shock was immediately preceded by a 60 -sec visual CS. Then, during two recovery sessions, the lever was again present and the rats could again earn food by pressing it. Suppression of leverpressing, relative to the last baseline training session, was the measure of fear conditioned to the context. Baker et al. reported that rats that had received unsignaled footshocks showed greater suppression than rats that had received signaled footshocks, which suggests that when the CS was a good predictor of the US, the context did not become strongly conditioned; that is, the CS overshadowed the context.

In contrast, our laboratory has reported a series of experiments in which a tone CS failed to overshadow contextual or temporal conditioning in conditioned defensive freezing in the rat (e.g., Williams, Frame, \& LoLordo, 1992). In Experiment 1 of Williams et al., four groups of rats experienced a single shock in each of five conditioning sessions. Shock was either signaled by a 30 -sec tone CS or was unsignaled, and shock onset occurred either 
120 or $1,200 \mathrm{sec}$ after the rat was placed in the apparatus. Conditioning was followed by two types of tests: First, defensive freezing to the CS and to temporal cues was examined in another context that had never been reinforced with shock. This test lasted 2 days, with the CS presented either 120 or $1,200 \mathrm{sec}$ after placement. In this test, rats froze in the stimulus-free intervals that matched the postplacement time at which shock had occurred in the prior conditioning stage. There was little freezing at other session times. Importantly, we found no evidence that signaling the US affected pretrial freezing. In a subsequent test, the rats were returned to the original conditioning context, and freezing was assessed for 1-min observation periods (60-120 and 1,140-1,200 sec after the rat was placed in the chamber). As in the first test, high levels of freezing were observed when the test interval matched the US time in conditioning, but we also observed freezing early in the session in the group that had been conditioned at 1,200. For Group 1,200, the freezing observed at the 120 -sec test interval can only be attributed to the formation of a context-US association; this group had never experienced shock at the 120 -sec time. Critically, the results of this second test in the original conditioning context also revealed no evidence of overshadowing. Rats that had been given signaled shocks during conditioning did not freeze less in any of these observation periods than those given unsignaled shocks. Using 15 sessions of conditioning, Experiment 2 replicated the null effects of the signaling manipulation in Experiment 1.

Although there are many procedural differences between the experiments of Baker et al. (1981) and Williams et al. (1992), including the use of multiple USs versus a single US per session and different measures of fear, perhaps the most likely cause of the discrepant findings is the use of a fixed time between the rat's placement into the chamber and presentation of the US by Williams et al., versus a variable time to the first US presentation and a variable intertrial interval (ITI) between successive US presentations, in the experiment of Baker et al. Consistent with this possibility, Balsam and Gibbon (1988) found no overshadowing of context by an auditory CS in pigeon autoshaping with a fixed time between food USs. On the other hand, Odling-Smee (1975a, 1975b) and Marlin (1981) found that a CS overshadowed context in fear conditioning with a variable ITI.

The aim of the present experiments was to determine whether, in a procedure otherwise like that of Williams et al. (1992), a more unpredictable US presentation time would allow the CS to overshadow the context. The conditioning parameters of Experiment 1 were similar to those used by Williams et al. (1992). A single footshock US was delivered at a fixed or variable time after the rat was placed in the context, and the US was either signaled or unsignaled. In a second experiment, we used conditioning parameters that more closely approximated those of Baker et al. (1981). A larger number of USs, separated by either fixed or variable ITIs, were scheduled in each of nine conditioning sessions, and the context was then tested for its ability to control behavior in the signaled and unsignaled groups.

\section{EXPERIMENT 1}

In Experiment 1, we asked whether signaling a footshock US with a tone CS would interfere with contextually evoked freezing if placement in the context provided little information about when the shock would occur. Half of the rats of each of the signaled and unsignaled groups received the US at a fixed time after placement in the chamber, and the other half received the US at a variable time after placement in the chamber.

\section{Method}

Subjects. The subjects were 48 experimentally naive male SpragueDawley rats obtained from Charles River, Canada. The rats ranged between 350 and $400 \mathrm{~g}$ in weight. They were maintained on an adlib food and water schedule and were housed in individual wire-mesh cages in a colony room on a 16:8-h light:dark cycle. Experimental sessions occurred during the light portion of the cycle.

Apparatus. The apparatus was the same as that used by Williams et al. (1992). Two distinctively different contexts were used. Context 1 (conditioning apparatus) was a white rectangular box $(58 \mathrm{~cm}$ long $\times 19 \mathrm{~cm}$ wide $\times 18 \mathrm{~cm}$ tall). The floor consisted of 2.5 mm diameter stainless steel bars spaced $1.5 \mathrm{~cm}$ apart. These bars allowed the presentation of a 1.0-sec, 1.0-mA shock, delivered by a Grason-Stadler shock generator and scrambler (Model E1064GS). The box sat on the floor of the experimental room and was brightly illuminated by a 40-W, 120-volt (AC) lamp.

Context 2 (test apparatus) was located in an opened soundattenuating box that was dimly illuminated by a $25-\mathrm{W}, 120-\mathrm{V}$ (AC) directed red light source located $1 \mathrm{~m}$ from the cube. The red light provided ample illumination for video recording, but was nearly invisible to the rats. Context 2 was a standard operant chamber, measuring $23 \mathrm{~cm}$ long $\times 20 \mathrm{~cm}$ wide $\times 20 \mathrm{~cm}$ tall, with clear acrylic sides and ceiling. The stimulus panel, rear wall, and floor tray were aluminum. The floor consisted of 4-mm stainless steel bars spaced $1.9 \mathrm{~cm}$ apart.

The same room housed both of the apparatuses. A speaker that could provide a $1500-\mathrm{Hz}, 82-\mathrm{dB}(\mathrm{C}$-scale) tone hung from the ceiling of the room. The animal's behavior was monitored remotely by a Panasonic WV-1550 low-light video camera that was positioned $1 \mathrm{~m}$ away from each apparatus. All sessions were videotaped. An Apple IIe computer located in an adjacent room controlled experimental events.

Behavior. The primary data were the percentages of sampled behavior that contained a freezing response in the pretrial and trial periods. A time sampling technique was used to measure freezing. Once every $5 \mathrm{sec}$ during the recording periods, when the pacing signal occurred on the video playback, the rat was scored as either freezing or not. Freezing was defined as the absence, over the next $0.25 \mathrm{sec}$, of any visible movement of the body and vibrissae except that required in order for respiration to occur. Videotapes were later rescored by an independent observer as a check on the primary observer's reliability. The 2 nd observer was not informed about group membership. Interobserver reliability was calculated at $92.8 \%$ by dividing the total number of agreements by the total number of samples $(N=540)$. The primary observer's data were used for subsequent analysis; they were transformed into a percentage and analyzed with a repeated measures analysis of variance (ANOVA) using a $p<.05$ rejection criterion. Included as between-subjects factors were signaling (CS vs. no CS groups) and schedule (fixed vs. variable). 
Depending on the particular analysis, the within-subjects factors were session and interval (the time of the observation after placement).

Procedure. The rats were first placed once into each of the two contexts for 5 min to allow them to become familiar with them. The order of context exposure was counterbalanced across the two days of this phase.

The two variables manipulated in the subsequent conditioning stage were (1) signaled versus unsignaled shock and (2) a fixed versus variable time to US presentation. There were four groups $(n=$ 12): Group VCS (a variable time US preceded by a CS), Group FCS (a fixed time US preceded by a CS), Group VnoCS (a variable time US not preceded by a CS), and Group FnoCS (a fixed time US not preceded by a CS). Conditioning lasted six sessions (Days 3-8). For the fixed groups, a single US was always presented $600 \mathrm{sec}$ after placement into the conditioning context, with the US either immediately preceded by a 30 -sec tone CS (FCS group) or not (FnoCS group). For the variable groups- the VCS and VnoCS groups- the US was also presented once per session, but $60,120,400,800,1,020$, or 1,200 sec after placement in the context. Each of the six placementshock intervals was experienced by each rat over the course of the six conditioning sessions, which resulted in a mean presentation time of $600 \mathrm{sec}$ across sessions. The order in which the intervals were presented was counterbalanced using a Latin square procedure; 2 rats in each of the VnoCS and VCS groups received one of the six sequences of intervals specified by the Latin square. All rats were removed from the chamber $90 \mathrm{sec}$ after the occurrence of shock. Thus, session length was fixed for Groups FCS and FnoCS, but it varied for Groups VCS and VnoCS.

In the CS groups, freezing was recorded during the 30 -sec trial period, as well as in the 30 -sec period prior to CS onset (pretrial). Freezing was recorded during the corresponding intervals in the noCS groups.

In order to examine control of freezing by temporal cues and the auditory CS in the absence of ceiling effects resulting from strong contextual control, rats were placed in the previously unreinforced test context and freezing was observed during a 30-sec pretrial period and a subsequent 30 -sec CS (the trial period). The CS began 90,570 , or $1,170 \mathrm{sec}$ after the start of the session in three separate tests, one on each of Days 9-11. The order of testing of the three intervals was counterbalanced; 2 rats in each group were tested with each of the six possible orders of the three test intervals. The rats were removed $90 \mathrm{sec}$ after the offset of the CS. We assumed that handling and placement in the conditioning context resulted in internal stimuli that varied over time and to which conditioning could occur. When we discuss temporal cues, we are referring to such stimuli. Handling and placement in the other context should have many stimuli in common with handling and placement in the conditioning context, and so there should be considerable generalization between the temporal stimuli in the two contexts.

On Day 12, in order to assess control of freezing by contextual cues, the rats were returned to the original conditioning context, and freezing was measured during the 60-120, 540-600, and 1,140 $1,200 \mathrm{sec}$ intervals. No CSs or USs were presented.

\section{Results and Discussion}

Conditioning. All groups came to anticipate the delivery of shock. The main finding was that, if anything, acquisition of pretrial freezing was more rapid, and not less, in the FCS and VCS groups than in the FnoCS and VnoCS groups (see top panel of Figure 1). A $2 \times 2 \times 6$ repeated measures ANOVA on the pretrial data revealed a main effect of signaling $[F(1,44)=4.31]$, which was the result of more freezing overall in the CS groups than in the no-CS groups, and a main effect of session $[F(5,220)=$ 55.20], which was caused by the acquisition of defensive freezing. There were no other main effects or interactions. The fact that higher rather than lower levels of pretrial freezing were evident in the CS groups relative to their no-CS counterparts indicates that pretrial freezing was not overshadowed by the CS. Additionally, we found no evidence, using the one trial per session procedure, that fixed and variable placement-shock intervals supported different levels of freezing.

The bottom panel of Figure 1 shows the data from the trial period. Because no CS occurred during this period for the FnoCS and VnoCS groups, the freezing occurring during this time can be regarded as a control level for assessing the extent to which the tone augmented freezing in the FCS and VCS groups. As shown in Figure 1, conditioned freezing to the tone emerged quickly in the FCS and VCS groups, as indicated by a sharp increase in freezing between Sessions 1 and 2. This reflects freezing conditioned to the tone above any freezing conditioned to temporal and contextual cues. A $2 \times 2 \times 6$ repeated measures ANOVA confirmed this interpretation by revealing main effects of signaling $[F(1,44)=5.09]$ and session $[F(5,220)=31.75]$ and a signaling $\times$ session interaction $[F(5,220)=6.21]$. Further examination of the signaling $\times$ session interaction revealed more freezing in the FCS and VCS groups than in the FnoCS and VnoCS groups on Sessions $2[F(1,44)=21.73]$ and 3 $[F(1,44)=8.31]$. Freezing to the tone reached asymptotic levels on Session 3 in the FCS and VCS groups, and these groups did not differ from their respective no-CS groups thereafter, since baseline levels of freezing increased in the no-CS groups.

CS test. The data of the CS test shown in Figure 2 are organized in order to aid comparisons between the CS and no-CS groups. The top panel shows freezing in the fixed time groups, and the bottom panel shows freezing in the variable time groups as a function of the time of CS onset in test $(90,570$, or $1,170 \mathrm{sec}$ ) and period (pretrial and trial). The data most critical for the experimental hypothesis are the comparisons between the CS groups and their no-CS counterparts in the pretrial periods.

A $2 \times 2 \times 3$ repeated measures ANOVA on the pretrial data of the CS test revealed a different pattern of freezing across intervals (90-, 570-, and 1,170-sec) as a function of schedule (fixed vs. variable), which produced an interaction of schedule and interval $[F(2,44)=13.88]$. However, we did not find any main effects or interactions involving the signaling variable, nor were there any other main effects or interactions. These null results for the signaling variable are consistent with the data of the acquisition stage, which also revealed no evidence of lower pretrial freezing in the CS groups than in the no-CS groups.

Further analysis of the schedule $\times$ interval interaction revealed that freezing was maximal at the training time in the fixed time schedule, indicating that temporal conditioning occurred and transferred across contexts. The level of freezing in the pretrial period was highest at 540 $570 \mathrm{sec}$ after placement, lowest at 60-90 sec, and intermediate at $1,140-1,170 \mathrm{sec}$. This produced an increas- 


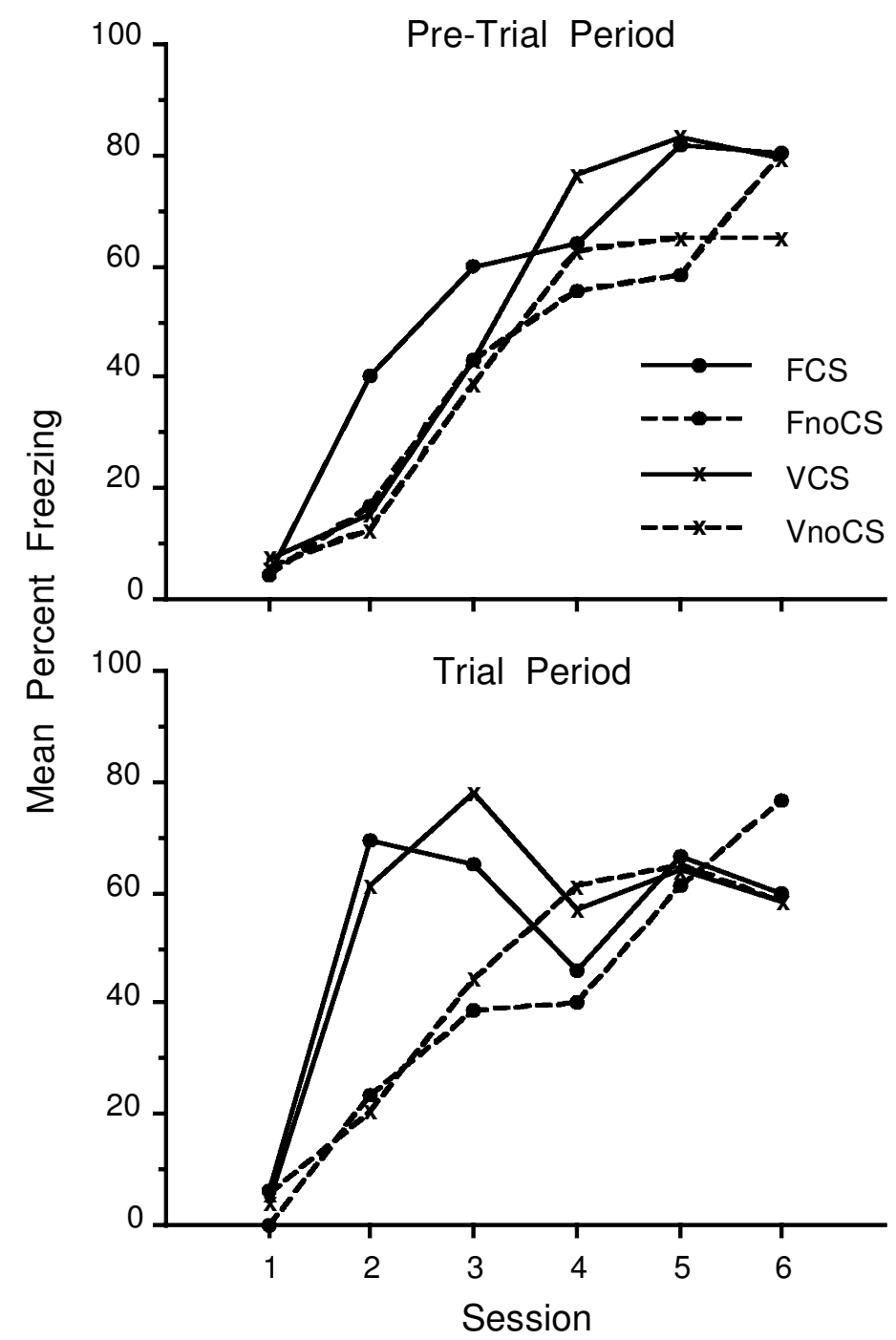

Figure 1. Mean percentages of samples scored as freezing in the pretrial and trial periods during the six conditioning sessions of Experiment 1. Rats were presented with the unconditioned stimulus (US) at a fixed time after placement into the context in Groups FCS and FnoCS or at a variable time after placement in Groups VCS and VnoCS. In the conditioned stimulus (CS) groups (FCS and VCS), the US was signaled by a 30-sec tone, whereas in Groups FnoCS and VnoCS, no CS occurred. Freezing during the pretrial period reflects the combined control of the context and time cues. In the CS groups, freezing in the trial period reflects the combined effects of context, time, and tone CS. In the no-CS groups, the CS was not presented, and so performance during the trial period is a resampling of control by context and time. FCS, fixed, signaled; FnoCS, fixed, unsignaled; VCS, variable, signaled; VnoCS, variable, unsignaled.

ing linear trend $[F(1,44)=7.50]$ and a quadratic trend $[F(1,44)=11.09]$ across observation intervals for the combined FCS and FnoCS groups. Thus, the rats in the FCS and FnoCS groups were not especially afraid of the context in which the CS test occurred; they were most afraid at the time, relative to placement in the context, at which they had previously experienced shock. Williams et al. (1992) observed analogous effects in groups of rats given other fixed placement-footshock intervals. For groups in the present experiment that had experienced USs at variable postplacement times, the VCS and VnoCS groups, pretrial freezing declined in a linear fashion over the test session $[F(1,44)=10.70]$.

A brief comment should be made about the data of the trial period, which are also shown in Figure 2. As expected, a $2 \times 2 \times 3$ repeated measures ANOVA revealed that higher levels of freezing were evoked by the tone in the signaled groups - Groups FCS and VCS - than in the 


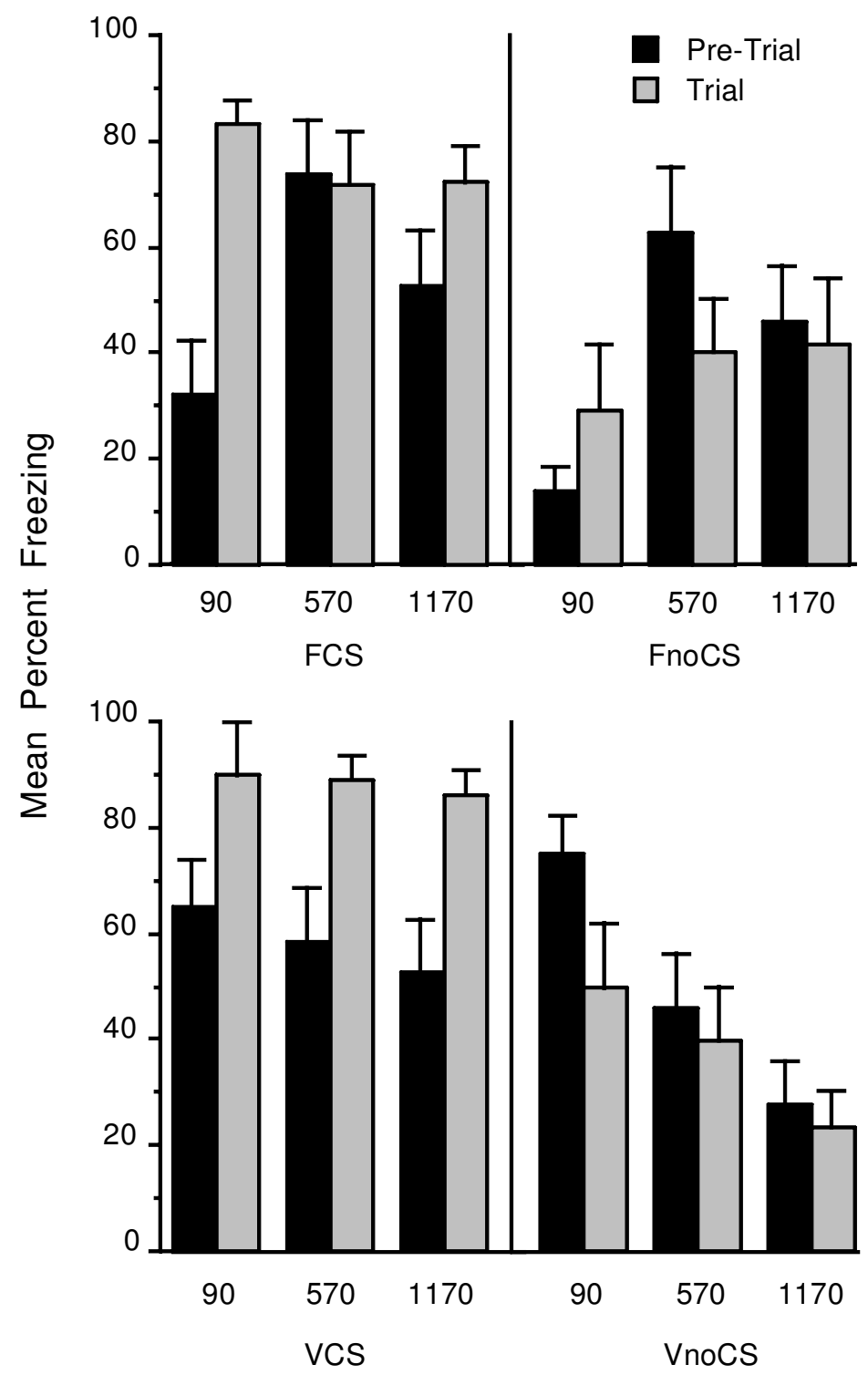

Figure 2. Mean percentage of samples in which freezing was observed during the conditioned stimulus (CS) tests in the unreinforced context for rats in the fixed time (FCS, FnoCS) and variable time (VCS, VnoCS) groups of Experiment 1. Each rat received the CS during the trial period, 90-120 sec after placement in the chamber in one session, 570-600 sec after placement in a second session, and 1,170-1,200 sec after placement in a third. The data are shown separately for the three testing times as a function of the $\mathbf{3 0}$-sec periods (pretrial and trial periods). FCS, fixed, signaled; FnoCS, fixed, unsignaled; VCS, variable, signaled; VnoCS, variable, unsignaled.

unsignaled groups, Groups FnoCS and VnoCS $[F(1,44)=$ 41.92]. There were no other main effects or interactions. The tone had previously been reinforced in the CS groups and was novel in the no-CS groups. Taken together, these data and those of the conditioning stage indicate that the null effects observed in the pretrial period cannot be explained away by suggesting the tone CS had not become strongly excitatory, and therefore, had little opportunity to interfere with temporal conditioning.
Context test. When the rats were returned to the original context of conditioning for a test in the absence of the CS and US (Figure 3), similar levels of freezing were observed in the FCS and FnoCS groups, and in the VCS and VnoCS groups, in all three test intervals (60-120, 540-600, and 1,140-1,200 sec). A $2 \times 2 \times 3$ ANOVA revealed only a main effect of interval $[F(2,88)=13.89]$ and an interaction of schedule and interval $[F(2,88)=$ 3.96]. Thus, these data confirm the findings of prior stages 


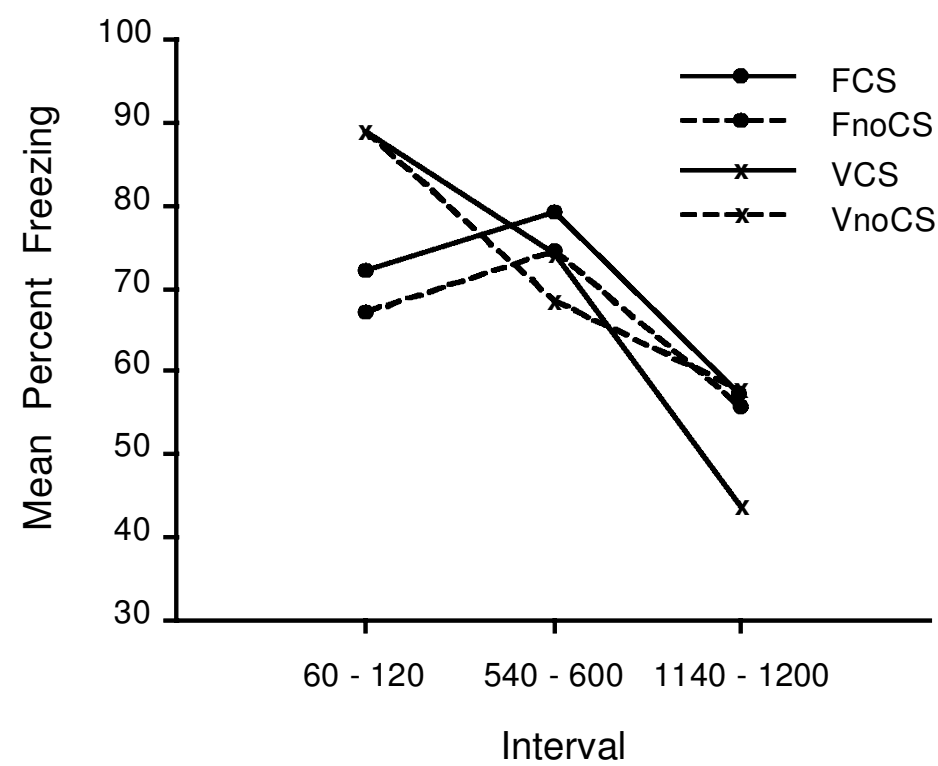

Figure 3. Freezing during the context test for rats in the fixed time and variable time groups of Experiment 1. Freezing was assessed during the 60 -sec intervals beginning 60,540, and 1,140 sec after placement in the chamber. The test occurred in the original conditioning context on the day after the last conditioned stimulus (CS) test. FCS, fixed, signaled; FnoCS, fixed, unsignaled; VCS, variable, signaled; VnoCS, variable, unsignaled.

that signaling the shock with the tone failed to attenuate conditioning to contextual cues in the VCS group and to temporal and contextual cues in the FCS group.

Again, in the context test, we found a different pattern of freezing in the fixed and variable time groups as a function of observation interval, which produced the interaction of schedule and interval. There was a marginally significant linear decline in freezing as the session progressed in the FCS and FnoCS groups $[F(1,44)=$ $3.73, p<.06]$, and the data were quadratic in form $[F(1,44)=4.07]$. In the fixed time groups, some freezing at the early test time would be expected because fear conditioned to context would be present, but there should be no conditioned fear to the early temporal cues because shock had never been presented at the early time. At the 540-600 sec interval, there should be considerable conditioned fear to time cues, and perhaps a decrease in fear as a result of the contextual cues having undergone some extinction. The quadratic trend is compatible with both of these assumptions. By contrast, in the VCS and VnoCS groups there was only a sharp linear decline in freezing during the context test $[F(1,44)=26.91]$. In the VCS and VnoCS groups, contextual conditioning may simply have extinguished over the unreinforced test session.

\section{EXPERIMENT 2}

Maes and LoLordo (1996) found no evidence of overshadowing of contextual freezing after a single session in which 10 footshocks, each preceded by a 10 -sec CS, were presented at variable ITIs. Similarly, an unpublished experiment by the present authors revealed no overshadowing of contextual freezing by a 30-sec auditory CS when eight trials were given at variable ITIs in one session. Odling-Smee (1978b) reported that with variable ITIs, overshadowing may be hard to detect at moderate shock intensities without a large number of conditioning sessions. For this reason, Experiment 2 included nine conditioning sessions with eight trials per session. The experiment was a $2 \times 2$ factorial design that included signaled or unsignaled shocks and fixed or variable ITIs. The VnoCS and VCS groups experienced variable intervals from placement to the first US, between successive USs, and from the last US to removal. All intervals in the FnoCS and FCS groups were a constant 309 sec.

\section{Method}

Subjects and Apparatus. The subjects were 32 experimentally naive male Sprague-Dawley rats (8 per group) obtained from Charles River, Canada. The same two distinctive contexts used in Experiment 1 served as the conditioning and test contexts. The CS and US were identical to those of Experiment 1.

Procedure. On Day 1, the rats were familiarized with the conditioning context. On Day 2, subjects were exposed to Context 2, which was not used again until the last day of the experiment for the CS test. No events were programmed during these 5-min sessions.

Pavlovian conditioning occurred on Days 3-11. Eight footshocks were presented in each session. In the VCS and VnoCS groups, there were variable intervals between placement in the chamber and the first shock, between successive shocks, and between the last shock and removal from the chamber. These intervals were randomly drawn without replacement from the following list: $34,76,112$, $153,204,267,352,483$, and $829 \mathrm{sec}$. Each rat was exposed to a different random sequence in each session. Rats in the FCS and FnoCS 
groups received a fixed ITI that was the arithmetic mean of the intervals used in the variable ITI groups (i.e., $309 \mathrm{sec}$ ). The time between placement and the first shock and the time between the last shock and removal were also $309 \mathrm{sec}$. Again, shocks were signaled in the VCS and FCS groups, but not in the VnoCS and FnoCS groups. Freezing was not assessed during conditioning, unlike in Experiment 1, because of the high density of shock USs.

On Days 12-14, we assessed the level of context conditioning. Freezing was recorded in Context 1 during five 1-min postplacement time intervals $(60-120,540-600,1,140-1,200,1,740-1,800$, and 2,340-2,400 sec). Rats were removed from the chambers $60 \mathrm{sec}$ after the last observation period. Thus each daily session was $41 \mathrm{~min}$ long. No CSs or USs were presented during these sessions. This assessment of context conditioning occurred 1,2, and 3 days after conditioning because, unlike in Experiment 1, contextual control had not been assessed during the conditioning sessions.

On Day 15, the effects of the auditory CS were assessed in Context 2. All four groups received five presentations of the 30 -sec tone CS. Freezing was recorded during 1-min intervals (60-120, $540-600,1,140-1,200,1,740-1,800$, and 2,340-2,400 sec). The tone CS occurred in the last $30 \mathrm{sec}$ of each recording period; the first $30 \mathrm{sec}$ of the recording period was the pretrial period. In Experiment 2, after the experiment was completed videotaped sessions were scored by an independent observer who was blind to the experimental treatment of the animal.

\section{Results and Discussion}

Context test. The major finding of the present experiment was that, after many sessions of conditioning with many trials per session, we found overshadowing of freezing in the variable ITI condition but not in the fixed ITI condition. Figure 4 illustrates the mean percent freezing for each of the 1-min observation intervals of the context test. The figure shows a large overshadowing effect in the variable ITI condition, with significantly more freezing in the VnoCS group than in the VCS group. A $2 \times 2$ $\times 3 \times 5$ repeated measures ANOVA on these data revealed main effects of schedule $[F(1,28)=5.42]$, session $[F(2,56)=25.78]$, and interval $[F(4,112)=11.32]$, and interactions of session and interval $[F(8,224)=2.43]$ and of signaling $\times$ schedule $\times$ session $\times$ interval $[F(8,224)=2.00]$. Of most interest is the four-way interaction that was caused by variations in the magnitude of the overshadowing effect in the variable ITI groups across intervals and successive test sessions. Significant differences between the VCS and VnoCS groups were found on the first, fourth, and fifth intervals of Session 1 [smallest $F(1,28)=6.08]$ and the first and third intervals of Session 2 [smallest $F(1,28)=4.43$ ]. Consistent with Experiment 1 , there was no evidence of overshadowing for groups that received USs at regularly scheduled intervals. The level of freezing in the FnoCS and FCS groups was similar during each of the sampled intervals. In summary, Experiment 2 showed that with extended conditioning, there was overshadowing in the variable ITI con-

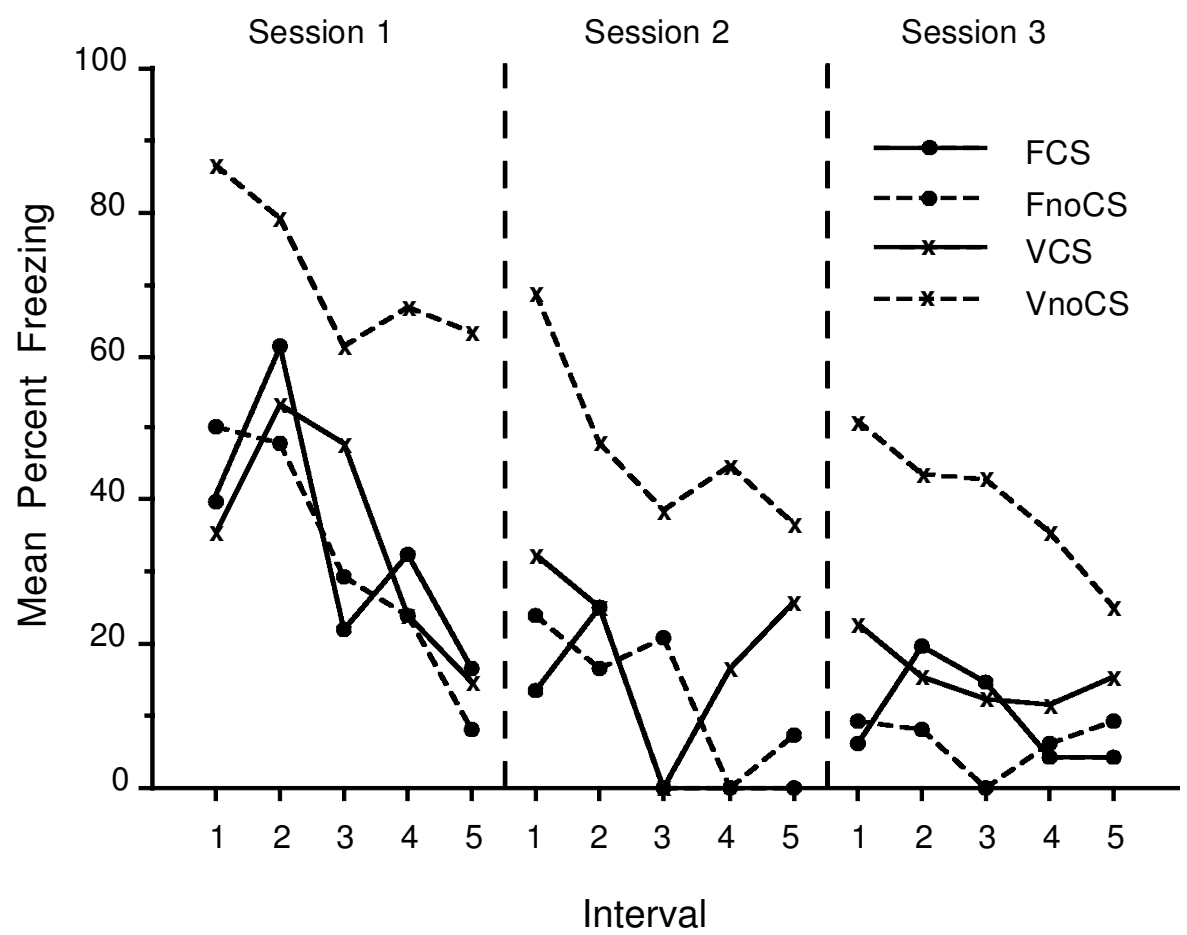

Figure 4. Freezing in the conditioning context for rats that had previously received signaled or unsignaled shocks at variable or fixed intervals for nine sessions in Experiment 2. On each of three daily sessions, freezing was assessed during the 60 -sec intervals from 60 to 120 sec (Interval 1), 540 to 600 sec (Interval 2), 1,140 to 1,200 sec (Interval 3), 1,740 to 1,800 sec (Interval 4), and 2,340 to 2,400 sec (Interval 5) after placement in the conditioning chamber. FCS, fixed, signaled; FnoCS, fixed, unsignaled; VCS, variable, signaled; VnoCS, variable, unsignaled. 
dition but not in the fixed ITI condition. As predicted, the temporal cue provided by the constant placement-US and US-US interval was not overshadowed.

These results suggest that Williams et al. (1992) may have failed to find overshadowing because their design allowed placement cues to signal the precise arrival time of the US. Why, in their Experiments 3 and 4, when the US was presented alternately at $120 \mathrm{sec}$ and 1,200 sec after placement, was there no overshadowing? It could be that scheduling the US at two times is not the same as having variable presentation time, especially given that temporal control has been observed to some extent after only a single conditioning trial (Maes \& Vossen, 1992).

Why did Experiment 2 show that variable ITIs supported higher levels of freezing to context than did fixed ITIs (in VnoCS vs. FnoCS)? The most likely explanation is that the rats learned something about when shock occurred. For example, the rats in Group VnoCS experienced both long and short US-free periods followed by reexposure to the US. Thus, it is possible that they learned that shock occurred at unpredictable intervals. If so, high levels of freezing would be expected throughout the test session. By contrast, in Group FnoCS the shocks were presented at constant intervals; thus, the rats in this group may have experienced an extinction trial when shock did not arrive at its usual time in test. The lower rate of freezing in Group FnoCS relative to Group VnoCS in the first observation interval can also be understood by assuming that the rats had learned something about the interval between placement in the context and the occurrence of the first US. Unlike Group VnoCS, Group FnoCS had never experienced a US during the first 2 min after placement in the conditioning context, the period that corresponds to the first observation interval.

Of course, it is always possible that the variable and fixed schedules affected overall levels of freezing for other reasons. For example, it is possible that Group VnoCS became sensitized to the shock because of a variable ITI. If so, there might have been less freezing in Group FnoCS than in Group VnoCS because a sensitized US could serve as an especially effective reinforcer. Ruling out this alternative account would seem to require an independent assessment of the vigor of the unconditioned scrambling reaction to shock (e.g., Fanselow, 1982) at the end of conditioning stage, a test that we did not conduct. To be meaningful, such a test would have to occur in a different context than that used for conditioning.

CS test. As expected, the data from the final CS test confirmed that the CS had become excitatory in the VCS and FCS groups. A $2 \times 2 \times 5$ repeated measures ANOVA on the pretrial data revealed no main effects or interactions of any of the variables. Freezing in the pretrial period was very low even in the first observation interval. A $2 \times 2 \times 5$ ANOVA on the data of the CS period revealed main effects of signaling $[F(1,28)=73.14]$ and interval $[F(4,112)=2.63]$, and an interaction of signaling and schedule $[F(1,28)=5.76]$. As expected, the CS groups froze more than their no-CS counterparts, and freezing in both the CS and no-CS groups declined over intervals. The signaling $\times$ schedule interaction was caused by greater difference in freezing to the tone CS between the FCS and FnoCS groups than between the VCS and VnoCS groups. Overall levels of freezing to the tone CS were $\mathrm{FCS}=80 \%$, FnoCS $=0 \%, \mathrm{VCS}=62 \%$, and $\mathrm{VnoCS}=16 \%$.

\section{GENERAL DISCUSSION}

The present experiments support the notion that when footshock USs occur at regular temporal intervals, rats quickly learn about the time of US presentation, and this learning is difficult to overshadow. Experiments 1 and 2 showed no evidence that pretrial freezing was lower in the FCS group than in the FnoCS group. On the other hand, at least with multiple sessions of many trials per session, contextually evoked freezing was overshadowed in otherwise identical circumstances when footshock USs were presented at variable intervals (Experiment 2). With variable intervals, pretrial freezing can only come under the control of contextual cues. Thus, the variable groups in Experiment 2 might have frozen more than the fixed groups because they could not learn the US's arrival time. They were not "better" conditioned. Because placement in the context did not tell the animal when the US would occur, pretrial freezing was overshadowed by a CS that signaled the precise arrival time of the US. Thus, the present experiments have identified a major factor that accounts for previous discrepancies in the literature. There was evidence of overshadowing only when USs were scheduled at variable times, and only when there were many sessions and many trials per session.

Experiments examining overshadowing in overlapping serial compounds of discrete CSs (CS1-CS2-US) provide similar data to those found in the fixed time conditions of the present experiments. In this procedure, CS1 onset is followed by CS2 onset, and the two CSs terminate with the onset of the US. Kehoe (1983), studying nictitating membrane conditioning in the rabbit, and Ayres (1966), investigating conditioned suppression in the rat, both found that CS2 failed to overshadow CS1. In this case, closer proximity of CS2 to the US would seem to make it a better predictor of the US's arrival time. However, CS1 marks the beginning of the trial, and it is the first event to signal the US. In the present experiments, placement of the rat in the conditioning context provided information that was not available from other cues present at that time. In the FCS and FnoCS groups, placement also signaled when the US would occur, as CS1 does in an overlapping CS1-CS2 compound.

In Experiment 1 there was a variable time between the rat's placement in the conditioning chamber and the occurrence of the US in the VCS and VnoCS groups. Unlike in Experiment 2, the presence of an excitatory CS had no effect on the level of freezing in the pretrial period. Perhaps performance in this instance was preasymptotic, and further conditioning would have revealed 
overshadowing of situational cues. Odling-Smee (1978b), using the context preference measure of conditioned fear, found that with a 1.3-mA US there was no overshadowing after 4 conditioning sessions. However, he observed substantial though incomplete overshadowing after 12 sessions. Moreover, in an experiment otherwise like Experiment 2, but with a single conditioning session, there was no overshadowing of situational cues by the discrete CS even with variable intervals between trials (see also Maes \& LoLordo, 1996). Whether more conditioning trials in the one trial per session procedure of Experiment 1 would have revealed overshadowing in the variable time groups remains to be seen. Conclusions based on comparisons between the two experiments reported here must be tempered because the experiments differed in several conditioning parameters other than the total number of trials (6 vs. 72). These included number of sessions (6 vs. 9 ), number of trials per session (1 vs. 8; but see above), and mean ITI (600 vs. $309 \mathrm{sec})$.

One possible explanation for why overshadowing might have emerged with extended conditioning is that cue competition would not normally begin until the CS has acquired a meaningful level of associative strength. However, our CS was always well conditioned and there was only a single case of overshadowing. In addition, Williams and LoLordo (1995) found no evidence that an already excitatory tone CS blocks the conditioning of temporal and contextual cues. A better account of our data is provided by discrimination theory. According to this theory, when signaled shocks occur at variable intervals, rats may gradually learn to be afraid of the context and the $\mathrm{CS}$, and not to be afraid of the context in the absence of the CS (McAllister, McAllister, Weldon, \& Cohen, 1974). If we assume that the VCS group receives a relatively large number of unreinforced context-alone trials for every reinforced presentation of the context-tone compound, then we would expect generalization of conditioned responding from the compound to context alone, followed by a decline in responding to context alone as it is repeatedly unreinforced. On the other hand, when the interval between placement in the conditioning chamber and the occurrence of footshock is fixed, as in Group FCS, there is no basis for discrimination because both time since placement in the chamber and time since the start of the CS are perfectly correlated with the occurrence of footshock.

Apparently at odds with the results of our experiments is a report by Maier and Keith (1987, Experiment 4; see also Jackson \& Minor, 1988), who observed that a brief visual signal strongly overshadowed context when rats received 100 intense tailshocks at fixed 60-sec intervals in a single session. Goddard (1995) found a similar effect when a visual stimulus preceded each of 150 food presentations that occurred at $60-\mathrm{sec}$ intervals. One hundred trials are more than we used in any of our experiments, and so it is possible that our outcomes with fixed ITIs are also preasymptotic. On the other hand, Balsam and Gibbon (1988) found that although increasing the number of food presentations at fixed intervals from 2 to 1,200 produced an increase in the associative strength of the context in which the food was presented, there was no overshadowing of context by an auditory CS even after 1,200 trials.

One issue of special concern is whether the overshadowing seen in Experiment 2 is a secondary consequence of baseline differences in freezing in the variable and fixed time groups. McAllister et al. (1974) found that as ITI duration increased from 165 to $285 \mathrm{sec}$ across groups, with the CS-US interval held constant at $4 \mathrm{sec}$, the absence of overshadowing was replaced by overshadowing. This outcome was largely attributable to greater context conditioning in the control group with an increased ITI. In the present experiments, it is quite unlikely that variable ITIs were having their effect by merely increasing baseline levels of freezing. There were similar and high levels of freezing in the VnoCS and FnoCS groups in Experiment 1 but no overshadowing. Additionally, in Experiment 2 of Williams et al. (1992) there were high levels of freezing in combination with a very large number of conditioning sessions but there was no overshadowing. In that experiment, the lack of overshadowing is best attributed to the fixed temporal placement of the US either early $(120 \mathrm{sec})$ or late $(1,200 \mathrm{sec})$ in the conditioning session.

In closing, it is worth calling attention to a shared implication of these data and those of an earlier experiment by Odling-Smee (1978a). Using a delay conditioning procedure, Odling-Smee found that a nominal CS would not overshadow context if CS duration varied from trial to trial $(7,13,33,65$, or $98 \mathrm{sec})$. In this case, the CS was associated with the US, but the time from CS onset to the arrival of the US was uncertain. An entirely different result was obtained with another group of rats that received a trace procedure with a variable duration CS. As in the delay procedure, CS onset did not predict the arrival time of the US. However, the offset of the trace CS was always followed $10 \mathrm{sec}$ later by the US, and this constant interval provided a precise temporal cue for the arrival of the US. Odling-Smee found that the level of overshadowing seen in the trace group trained with a variable CS duration was similar to the level of overshadowing seen in a delay group trained with a constant CS duration. Thus, the variable duration CS overshadowed situational cues only when it told the animal when the US would occur. Our results indicate that situational cues become vulnerable to overshadowing only when they predict the arrival time of the US with less reliability than the nominal CS.

\section{REFERENCES}

Ayres, J. J. B. (1966). Conditioned suppression and the information hypothesis. Journal of Comparative \& Physiological Psychology, 62, 21-25.

Baker, A. G., Mercier, P., Gabel, J., \& Baker, P. A. (1981). Contextual conditioning and the US preexposure effect in conditioned fear. Journal of Experimental Psychology: Animal Behavior Processes, 7, 109-128.

Balsam, P. D., \& Gibbon, J. (1988). Formation of tone-US associations 
does not interfere with the formation of context-US associations in pigeons. Journal of Experimental Psychology: Animal Behavior Processes, 14, 401-412.

Balsam, P. D., \& Tomie, A. (1985). Context and learning. Hillsdale, NJ: Erlbaum.

FANSELOW, M. S. (1982). The postshock activity burst. Animal Learning \& Behavior, 10, 448-454.

Gibbon, J., \& Balsam, P. (1981). Spreading association in time. In C. M. Locurto, H. S. Terrace, \& J. Gibbon (Eds.), Autoshaping and conditioning theory (pp. 219-253). New York: Academic Press.

Goddard, M. J. (1995). Acquisition of US-no US associations in Pavlovian conditioning. Learning \& Motivation, 26, 264-277.

JACKSON, R. L., \& MinOR, T. R. (1988). Effects of signaling inescapable shock on subsequent escape learning: Implications for theories of coping and "learned helplessness." Journal of Experimental Psychology: Animal Behavior Processes, 14, 390-400.

Jenkins, H. M., Barnes, R. A., \& Barrera, F. J. (1981). Why autoshaping depends on trial spacing. In C. M. Locurto, H. S. Terrace, \& J. Gibbon (Eds.), Autoshaping and conditioning theory (pp. 255284). New York: Academic Press.

Kenoe, E. J. (1983). CS-US contiguity and CS intensity in conditioning of the rabbit's nictitating membrane response to serial compound stimuli. Journal of Experimental Psychology: Animal Behavior Processes, 9, 307-319.

MAes, J. H. R., \& LoLordo, V. M. (1996). The effect of a discrete signal on context conditioning: Assessment by preference and freezing tests. Learning \& Motivation, 27, 428-450.

Maes, J. H. R. \& Vossen, J. M. H. (1992). One-trial aversive conditioning to contextual cues: Effects of time of shock presentation on freezing during conditioning and testing. Bulletin of the Psychonomic Society, 30, 403-406.

MaIER, S. F., \& KeITH, J. R. (1987). Shock signals and the development of stress-induced analgesia. Journal of Experimental Psychology: Animal Behavior Processes, 13, 226-238.

Marlin, N. A. (1981). Contextual associations in trace conditioning. Animal Learning \& Behavior, 9, 519-523.

McAllister, W. R., McAllister, D. E., Weldin, G. H., \& Cohen, J. M. (1974). Intertrial interval effects in classically conditioning fear to a discrete conditioned stimulus and to situational cues. Journal of Comparative \& Physiological Psychology, 87, 582-590.

Miller, R. R, \& Schachtman, T. R. (1985). The several roles of context at the time of retrieval. In P. D. Balsam \& A. Tomie (Eds.), Context and learning (pp. 167-194). Hillsdale, NJ: Erlbaum.

OdLing-Smee, F. J. (1975a). Background stimuli and the interstimulus interval during Pavlovian conditioning. Quarterly Journal of Experimental Psychology, 27, 387-392.

OdLing-Smee, F. J. (1975b). The role of background stimuli during Pavlovian conditioning. Quarterly Journal of Experimental Psychology, 27, 201-209.

ODLING-SMeE, F. J. (1978a). The overshadowing of background stimuli by an informative CS in aversive Pavlovian conditioning with rats. Animal Learning \& Behavior, 6, 43-51.

OdLING-SMeE, F. J. (1978b). The overshadowing of background stimuli: Some effects of varying amounts of training and UCS intensity. Quarterly Journal of Experimental Psychology, 30, 737-746.

Rescorla, R. A., \& Wagner, A. R. (1972). A theory of Pavlovian conditioning: Variations in the effectiveness of reinforcement and nonreinforcement. In A. H. Black \& W. F. Prokasy (Eds.), Classical conditioning II: Current research and theory (pp. 105-132). New York: Appleton-Century-Crofts.

WAGNeR, A. R, \& Rescorla, R. A. (1972). Inhibition in Pavlovian conditioning: Application of a theory. In R. A. Boakes \& M. S. Halliday (Eds.), Inhibition and learning (pp. 301-336). London: Academic Press.

Williams, D. A., Frame, K. A., \& LoLordo, V. M. (1992). Discrete signals for the unconditioned stimulus fail to overshadow contextual or temporal conditioning. Journal of Experimental Psychology: Animal Behavior Processes, 18, 41-55.

Williams, D. A., \& LoLordo, V. M. (1995). Time cues block the CS, but the CS does not block time cues. Quarterly Journal of Experimental Psychology, 48B, 97-116.

(Manuscript received July 5, 2000;

revision accepted for publication February 4, 2001.) 